\title{
Geriatric visiting hours: lessons for the general wards?
}

\section{N W Griffith}

Department of

Geriatric Medicine,

University College

Hospital, London

WCIE 6AU

D N W Griffith, MD, senior registrar
$B M F$ 1992;304:292

Ease of access for people's visitors when they are in hospital not only is a reasonable expectation but has also been shown to be beneficial and is recommended in longstanding government guidelines.' $\mathrm{A}$ previous survey documented that many restrictions remain in general medical and surgical wards. ${ }^{2}$ The present survey was undertaken to ascertain the position in departments of geriatric medicine.

\section{Survey and results}

Letters were sent to hospital managers in 760 hospitals requesting details of visiting in geriatric wards - that is, all hospitals listed in the Medical Directory with over 50 beds and with consultants in geriatric medicine on the staff.

Replies were received from $579(76 \%)$ of the hospitals. Several reported "open" visiting for a set number of hours a day, in which case this figure was used, the term "open" being reserved for those hospitals which had no restrictions. Categories of more than 5 hours/day, 2-5 hours/day, and less than 2 hours/day were used to allow comparison with the previous survey, and no subdivisions were made between two and five hours because of the small numbers. To investigate whether various types of hospitals set different limits on visiting, general, small, and non-acute hospitals were analysed separately (table), but no significant differences emerged, with all subgroups allowing over five hours a day in over $85 \%$ of instances.

Numbers (percentages) of hospitals ${ }^{\star}$ allowing various visiting periods on geriatric wards

\begin{tabular}{|c|c|c|c|c|}
\hline $\begin{array}{l}\text { Visiting hours } \\
\text { (hours/day) }\end{array}$ & All hospitals $\dagger$ & $\begin{array}{l}\text { General hospitals } \\
\quad(>200 \text { beds })\end{array}$ & $\begin{array}{l}\text { Small hospitals } \\
\text { ( } 50-100 \text { beds })\end{array}$ & $\begin{array}{c}\text { Non-acute hospitals } \\
\text { (no accident and emergency } \\
\text { department; mostly solely } \\
\text { geriatric or psychogeriatric) }\end{array}$ \\
\hline Open & $282(49)$ & $107(46)$ & $93(56)$ & $150(55)$ \\
\hline $6-12$ & $230(40)$ & $90(39)$ & $67(40)$ & $106(39)$ \\
\hline $2-5$ & $61(11)$ & $30(13)$ & $6(4)$ & $14(5)$ \\
\hline$<2$ & $6(1)$ & $4(1 \cdot 7)$ & $1(0 \cdot 6)$ & $2(0 \cdot 7)$ \\
\hline Total & 579 & 231 & 167 & 272 \\
\hline
\end{tabular}

* Some hospitals fall into more than one category of hospital

tIncludes general hospitals with only 100-200 beds.
The previous survey of general medical and surgical wards showed pronounced regional variations in visiting times, but data from the present survey showed no such pattern.

\section{Comment}

Hawker has outlined the change of emphasis in visiting arrangements from "for the good of the organisation" to "for the good of the patient." Many now recognise that these two objectives may coincide, and an extension of visiting times can help both patient and hospital. ${ }^{1245}$ That these lessons have been assimilated by those concerned in the care of elderly people seems to be borne out by the results of this survey.

This contrasts with the position in general wards. Whereas $88 \%$ of hospitals reported visiting for more than five hours a day for geriatric wards, only $33 \%$ did so for general wards. While only six hospitals $(1 \%)$ restricted visiting to less than two hours a day in geriatric wards, a quarter of all hospitals imposed such limits in medical and surgical wards. Although the surveys of general and geriatric wards were carried out at different times, information gained from the present survey, in which several hospitals supplied details for all wards, suggested that major differences still exist between geriatric and general wards.

How has such a gap developed? There seems no intrinsic reason why extended visiting should be a problem on general wards. Difficulties in patient care caused by visitors - an argument used to defend limited visiting-does not stand up. Firstly, there is no evidence that the nursing care and procedures necessary on geriatric wards are less time consuming and laborious than those carried out on general wards; the reverse may even be true. Secondly, and more importantly, about one third of the general medical and surgical wards previously surveyed already permitted liberal visiting. The onus now is surely on those who wish to perpetuate restricted visiting to justify their stance. It is difficult to see why extended visiting times should not now be the norm.

1 Department of Health and Social Security and Welsh Office. The organisation of the in-patients' day. Report of a Committee of the Central Health Services Council. London: HMSO, 1976

2 Griffith DNW. Hospital visiting hours: time for improvement. BMF 1988:296: $1303-4$

3 Hawker R. Rules to control visitors; 1746-1900. Nursing Times 1984;80:49-51.

4 Gawker R. Rules to control visitors; 1746-1900. Nursing Times 1984,80

5 Irving RE, Smith BJ. Patterns of visiting. Some experiences of free visiting in a general hospital geriatric unit. Lancet 1963;i:597-600.

(Accepted 27 September 1991
Department of Applied Biology, University of Hull, Hull HU6 7RX

Carolyn E Grundy, PHD, research assistant

John Robinson, PHD, senior lecturer

Princess Royal Hospital, Hull

Katharine A Guthrie, MRCOG, senior registrar Alan G Gordon, FRCOG, consultant gynaecologist Douglas M Hay, FRCOG, consultant gynaecologist and obstetrician

Correspondence to: Dr Grundy.

\section{Establishment of pregnancy after removal of sperm antibodies in vitro}

\author{
Carolyn E Grundy, John Robinson, \\ Katharine A Guthrie, Alan G Gordon, \\ Douglas M Hay
}

About $10 \%$ of infertile men have an immunological basis to their sterility. Sperm antibodies may be present on any part of the sperm surface, head, midpiece, or tail and the impairment of fertilising capacity depends on the percentage of sperm coated with these antibodies and the type of immunoglobulin present.' A synergistic combination of IgG and IgA is thought to be of particular clinical importance, but some workers report that IgA alone is sufficient to inhibit fertilisation. ${ }^{2}$ The only treatment currently available to alleviate this condition is systemic corticosteroids, but the efficacy is doubtful ${ }^{3}$ and treatment may produce unpleasant or unacceptable side effectsfor example, hip necrosis, exacerbation of incipient duodenal ulcers, cardiovascular effects. Previous attempts to remove sperm antibodies in vitro by washing and centrifugation have been shown to be ineffective, because of the high affinity of these immunoglobulins for sperm surface antigens: even washing up to 18 times is without apparent effect. ${ }^{4}$

Recently we devised a technique whereby sperm antibodies can be effectively removed from the sperm surface in vitro. ${ }^{5}$ We report the first applications of this technique to assisted conception procedures and the outcome - the establishment of two pregnancies.

\section{Patients, methods, and results}

The table shows the relevant clinical history of the three cases of immunological infertility treated by removal of sperm antibodies.

Semen was collected by masturbation and ejacula- 
tion directly into warm culture medium (Medicult, Copenhagen). Antibody free sperm were prepared as detailed previously. ${ }^{5}$ Briefly, this entailed processing on a discontinuous Percoll gradient followed by incubation with immunobeads (Biorad Laboratories, Watford) that had been washed twice in HAMS F-10 medium to remove sodium azide. Anti-IgA beads ( $3 \mathrm{mg}$ ) were used in case 1 and a mixture of anti-IgG and anti-IgA beads ( $3 \mathrm{mg}$ of each) in cases 2 and 3. The incubation was carried out with gentle but thorough mixing every 10-15 minutes at $37^{\circ} \mathrm{C}$ in an atmosphere of air containing $5 \%$ carbon dioxide. Warm in vitro fertilisation medium $(200 \mu \mathrm{l})$ was then carefully layered over the bead-sperm mixture and incubated under the same conditions for a further two hours. The supernatant was removed and the sperm were tested for the presence of antibodies with the direct immunobead test. The percentage of sperm shown to be antibody positive was reduced to less than $10 \%$ in cases 1 and 3 and to $10-15 \%$ in case 2 .

Sperm stripped of antibodies in this way were used

Clinical details of three cases of immunological infertility and outcome of removal of sperm antibodies on in vitro fertilisation

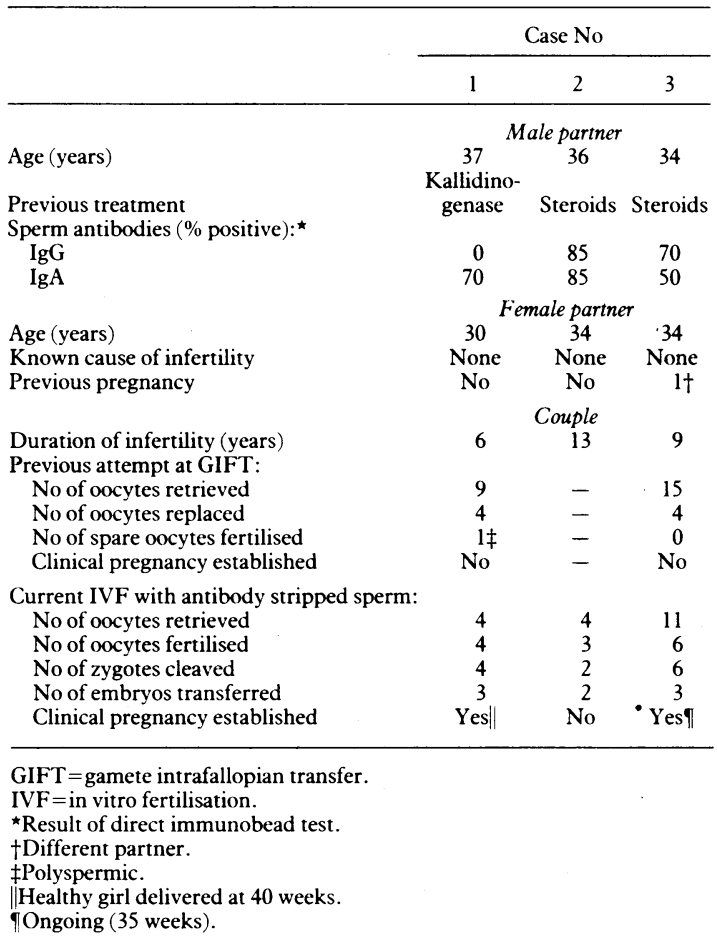

during treatment cycles of in vitro fertilisation; the laboratory and clinical outcome is shown in the table. The oocytes obtained in case 1 were of high quality and resulted in four excellent embryos. One of the oocytes recovered in case 2 was degenerate; one of the three embryos obtained degenerated after 24 hours but the two others were transferred and were of good quality. The oocytes obtained in case 3 were more variable and included several that were immature and did not fertilise; the three embryos that were transferred, however, were of good quality. Overall, two pregnancies were established (cases 1 and 3).

\section{Comment}

Despite the initially high levels of sperm antibodies present in the semen of the male partner in these three cases, all of the mature oocytes recovered from the female partner were successfully fertilised after the antibodies had been stripped in vitro. This outcome contrasts sharply with the data from the previous attempts at gamete intrafallopian transfer in cases 1 and 3. Although the other semen variables in case 2 were normal, fertilisation would probably not have occurred without the antibody stripping as the percentage of sperm coated with antibody of both isotypes was very high, as was the degree of sperm clumping. In this case two high quality embryos were transferred, although a pregnancy did not result.

This technique thus seems to be of clinical importance in the treatment of immunological infertility by in vitro fertilisation and other forms of assisted reproduction. It is effective, has none of the side effects of systemic steroids, and can be incorporated easily into the routine of a laboratory specialised in assisted reproduction techniques.

This work was funded by a grant from Yorkshire Regional Health Authority.

1 Bronson R, Cooper G, Rosenfeld D. Sperm antibodies: their role in infertility. Fertil Steril 1984;42:171-83.

2 Clarke GN, Lopata A, McBain JC, Baker HWG, Johnston WIH. Effect of sperm antibodies in males on human in vitro fertilisation (IVF). American fournal of Reproductive Immunology and Microbiology 1985;8:62-6.

3 Haas GG, Manganiello P. A double-blind, placebo-controlled study of the use of methylprednisolone in infertile men with sperm-associated immunoglobins. Fertil Steril 1987;47:295-301.

4 Haas GG, D'Cruz OJ, Denum BM. Effect of repeated washing on sperm-bound immunoglobin G. F Androl 1988;9:190-6.

5 Grundy CE, Robinson J, Gordon AG, Hay DM. Selection of an antibody-free population of spermatozoa from semen samples of men suffering from immunological infertility. Hum Reprod 1991;6:593-6.

(Accepted 8 October 1991 )

\section{ONE HUNDRED YEARS AGO}

\section{RARE TARSO-METATARSAL DISLOCATION}

A simple dislocation of the metatarsals without fracture of any of the bones of the foot or leg is an accident of so rare occurrence that I beg to record the following:-

On November 1st, 1891, Captain O. was out shooting mounted on a riding camel, when the animal bolted and fell down a bank. The rider managed partially to free himself, but the left foot became entangled in the stirrup iron and was crushed by the body of the camel rolling upon it. On examining the foot, it was found that the metatarsal of the great toe was dislocated at the tarsometatarsal joint in a direction upwards and outwards. The internal cuneiform was uninjured, and the dislocation was a simple one. The metatarsals of the second, third, and fourth toes were also dislocated from the tarsus in an upward direction, and on running the hand over the dorsum the ridge formed by the dislocated bones and the tendons was distinctly felt. The point of the great toe was itself directed inwards and downwards, and on placing the feet together it impinged on the lower surface of the interphalangeal joint of the opposite great toe. The reduction was effected by extension, but some difficulty was experienced in replacing the metatarsal of the second toe, probably owing to its anatomical peculiarity of position. A pad of wool and bandages sufficed to keep the bones in place, and the foot was later on put in a starched bandage, with good results.

I believe cases similar to the above have been recorded by Liston, Dupuytren, and Smith, but on account of the strong ligamentous supports, the accident must be an extremely rare one.

Probably only a soft heavy body, such as a camel, would cause a similar injury, and I believe in the cases noted by the above surgeons the rolling of a horse on the leg and foot was the cause of the accident.

Zhob, Baluchistan

J. Chaytor White, M.B., I.M.S., 12th Bengal Cavalry (BMF 1892;i:15) 